\title{
Beyond the Notion
}

\section{Govinda Sah 'Azad'}

\begin{abstract}
My recent art practice involves the ancient symbol of 108, an Asian astronomical number that is used for ritual and tantric enlightenment. It represents the complete universe, and expresses coincidences in astronomical measurements, involving the relative diameters of the earth, moon and sun as well as the distances between them.
\end{abstract}

My recent artworks are inspired by, and attempt to describe in simple ways, the movement of clouds: how clouds form in nature, how they arise in nature, how they move. My inspiration now comes from clouds and the universe, and I am trying to express through my art some ideas of life, earth and the universe.

This, in itself, is not new; many artists, philosophers and religions have also used clouds as motifs. Clouds have also been used in almost every religion as symbols of the power of god, of the division between heaven and earth, of nature and to embody visions of heaven and earth. And there are well-established connections between astronomy, religion and philosophy. For example, in my religion, Hinduism, we read or chant 108 times to complete the circle of worship. There is no significant role or any particular reason behind it, but in the modern age the number 108 is also linked to scientific measurements of distance and space. Thus, although the connections between religion and science are not always understood, they seem to reflect a deeper reality. They seem to show how our body, mind, nature and the universe are connected, though most of time we are unconscious of these strange connections. This mysterious number, for example, reveals a deep relationship within itself and with the entire universe. My paintings attempt to explore some of these deeper spiritual and mystical connections.

In this paper I would like to share some of the ideas which I use every day in my art practice. All my works are created from my imagination, sometimes from dreams as well as reflected signs from nature. I don't try to copy from nature or from photographs and I don't use direct references.

Govinda Sah “Azad', 'Beyond the Notion'. eds. Nicholas Campion and Rolf Sinclair, Culture and Cosmos, Vol. 16 nos. 1 and 2, 2012, pp. 421-427. www.CultureAndCosmos.org 
My art practice started with landscape, nature and the daily life of Nepal. At the beginning I used to spend hours and hours every day in the landscape. I don't think I need to explain about the beauty of Nepal's landscape. As a learner I never stopped too long on one subject, I always moved; I liked to paint different and new landscapes. I once completed a three-month, solo cycling tour around my country while painting, carrying canvases, colours, an easel, and stopping to paint every 8 to 10 hours - it was a wonderful experience! After my cycling tour I went trekking in the foothills of Mount Everest and painted several mountain paintings. But my mind was just attracted to the clouds. I remembered how several artists had experimented with the sublime beauty of mountains and realised through them that I had become fascinated by the clouds and their power.

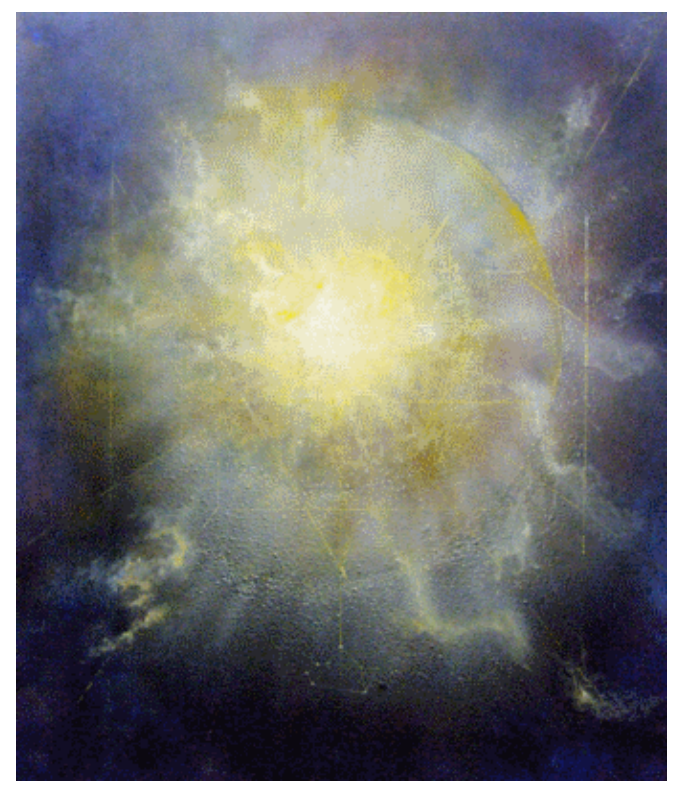

Figure 1. Between Present and Future, 2008, oil and acrylic on canvas, $120 \times 135 \mathrm{~cm}$

The clouds rose as steam from the mountain and, slowly and gradually as the temperature went up, they increased and covered the whole of the mountain. There was nothing for me to paint except those white clouds. I was overwhelmed; it was then I realised my experience of the sublime was not the world's tallest mountain: it was the clouds themselves. My 
first painting following this was a very different composition which I had never attempted before. The clouds had now become more powerful than either the light or the mountain. From 2004 to 2006 I went several times to Bangladesh; most of the paintings I made there were landscapes. Though Bangladesh is a flat land it is affected by the Himalayan winds and clouds. It was there that I finally started to paint clouds as my subject.

As a landscape painter, in many ways, I get inspiration from J.M.W. Turner and John Constable. These artists were also attracted to clouds as a subject matter and were inspired by them. I realised that clouds were not a different path for me, but my interpretation of them had to somehow evolve and become different. Clouds themselves have no colour, and consist of drops of water which, in turn, represent their own small universe. Clouds can thus suggest infinitely large and infinitely small distances. They can also represent a strong sense of light and dark, and of movement. Infinity lies behind the clouds, which I am trying to suggest in my paintings as an infinite universe and invisible space portrayed by visible clouds.

In Figure 2 I try to express those feelings of the drops of water and how they are carried out on a wave. For this work I used oil and acrylics. This was the beginning of my new form of artwork, inspired by Leibniz:

Reality cannot be found except in One single source, because of the interconnection of all things with one another. ... I maintain also that substances, whether material or immaterial, cannot be conceived in their bare essence without any activity, activity being of the essence of substance in general. ${ }^{1}$

One can see the effect on painting as similar to how drops of water give a three-dimensional feeling. The textures and the effect of light play a key role in my paintings. Working full time in the studio gives me enough time to develop those ideas more. Due to the infinite sky, my simple drops of water can easily be interpreted as the universe, or some distant planets or galaxies. Until recently I wasn't aware of the idea that my clouds could be interpreted as these things.

${ }^{1}$ G. W. Leibniz, New Essays on Human Understanding, abridged edition, Peter Remnant and Jonathan Bennet (ed and trans.) (Cambridge: Cambridge University Press, 1982), p. 66; 'The Dynamic Unity of Reality' at http://www.spaceandmotion.com (accessed 1 October 2010).

Culture and Cosmos 


\section{Beyond the Notion}

I encountered pictures from the Hubble Telescope when several friends suggested that I have a look. It was a really big surprise. The similarity of my inner imagination and the images from the Hubble pictures were not so very different. I was really moved with the idea, and could not find any logic about this similarity; I was puzzled. From there, I returned to my emotional ideas rather than make the same kind of pictures that were no different from those of Hubble's. This infinite subject I compared with our infinite desire and ambition. As one's life span is not long enough to fulfill one's own dreams and ambitions, so did I compare that to travelling to another planet.

To travel between one planet and another is not possible to achieve in a single human lifetime; the distances are too great. This knowledge brought a new movement into my art form that shifted from two-dimensional paintings into three-dimensional paintings. I created the painting, The Trap Within, in 2009; this was the first painting that I created in this way (Figure 2).

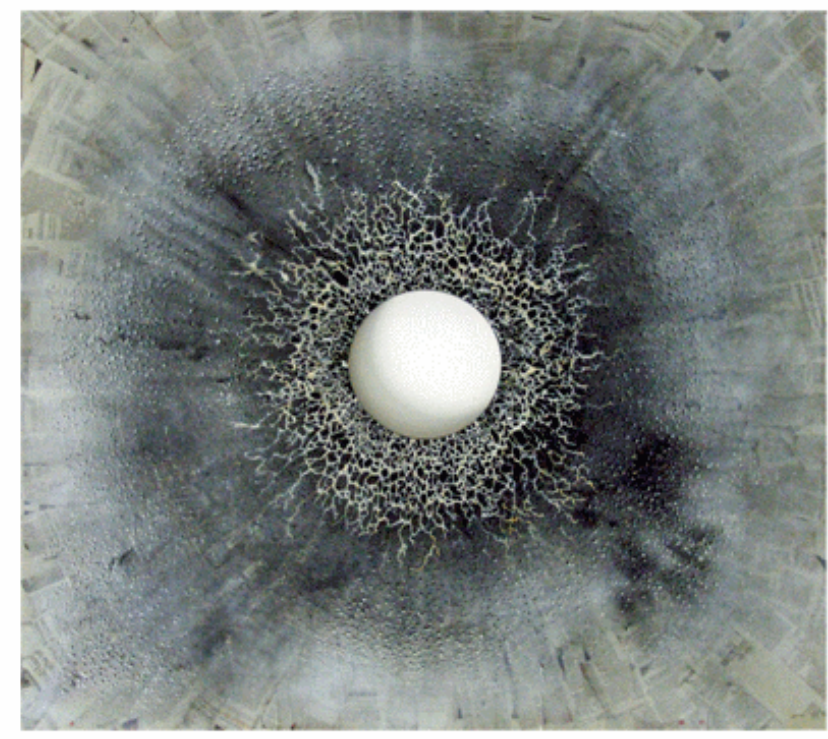

Figure 2. The Trap Within, 2009, oil, acrylic, spray and collage on canvas

The door was open for me to interpret how our life is tiny between these two infinities. Life is lost and the value of ambitions is empty to me; that was the reason for the hole in the centre of my paintings: that we can 
reflect upon the universe, which has no centre and has no edge. The hole in the middle of my paintings also became part of space and the reality of what kind of space that was, and the wall which can be seen behind.

A couple of paintings allowed me to look back and think about what I had been spending time on. Here I would like to share one of my ambitious works, a 5 by 2 metre triptych painting. It was created for my MA degree show (Figure 3).

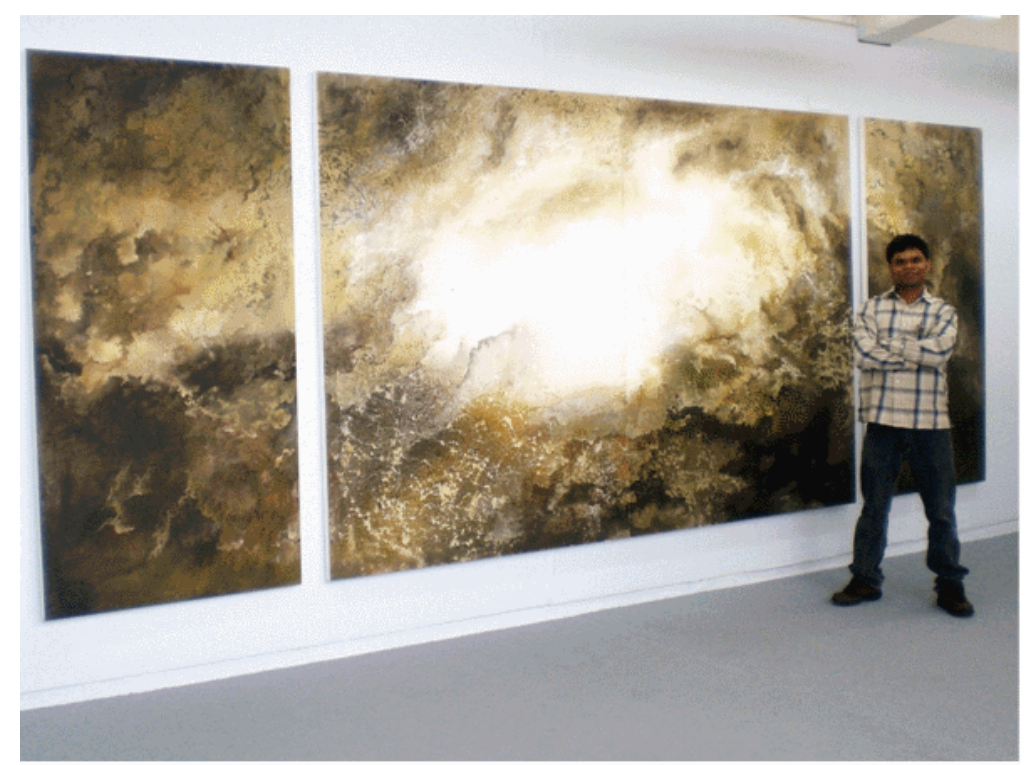

Figure 3. Hope and Horror - $I V$, 2008, acrylic on canvas, Collection in Landmark art, UK

Through my art I try to express how the universe feels. Everything is connected; as I breathe, the sun and space all appear to be breathing and moving. Nothing is separate; though space in between allows us to move around, every single object has its own gravity, which holds together the entire universe. These thoughts and questions can be solved easily in science classes, but in the studio you have to work with colours and canvases. The work I created in 2009 with the Trap Within and the Spiritual Gravity allowed my emotional reflection to realise these paintings. 


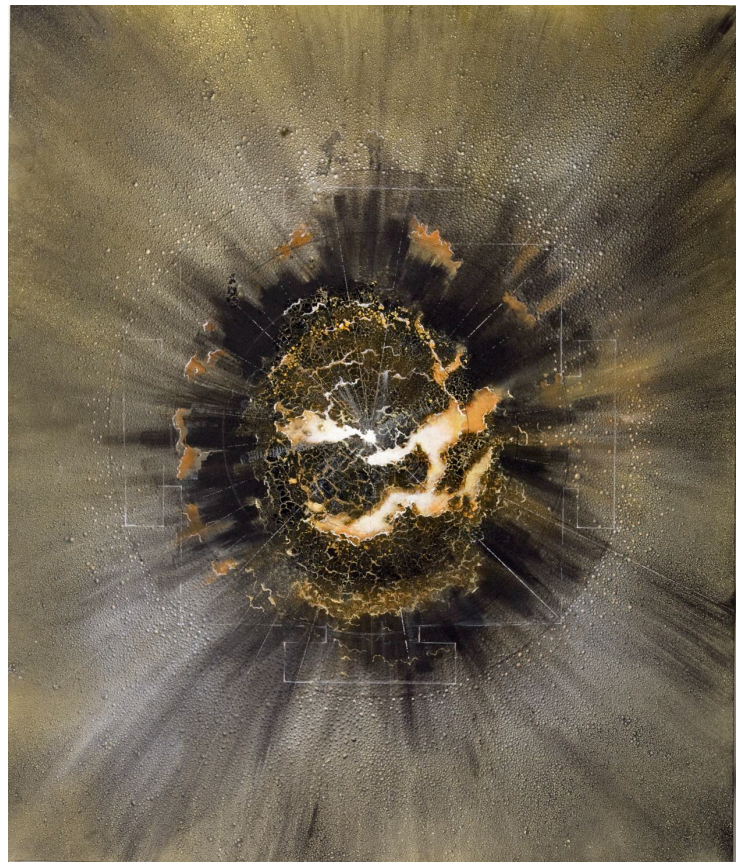

Figure 4. Despair Truth, 2010, oil and acrylic on canvas, 110 X $97 \mathrm{~cm}$

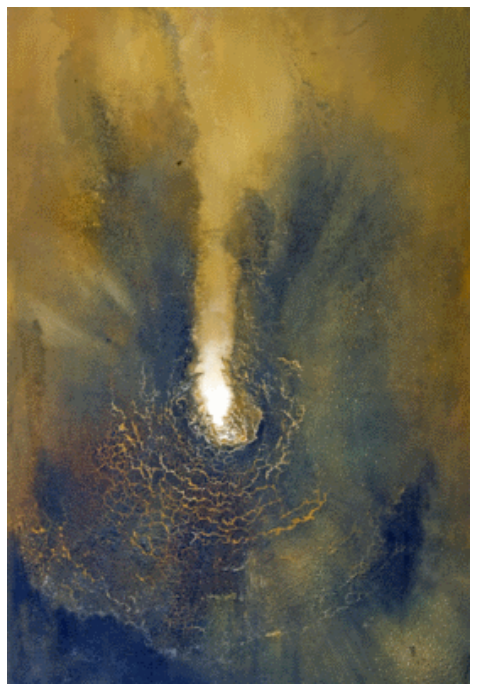

Figure 5. Birth of a Star, 2010

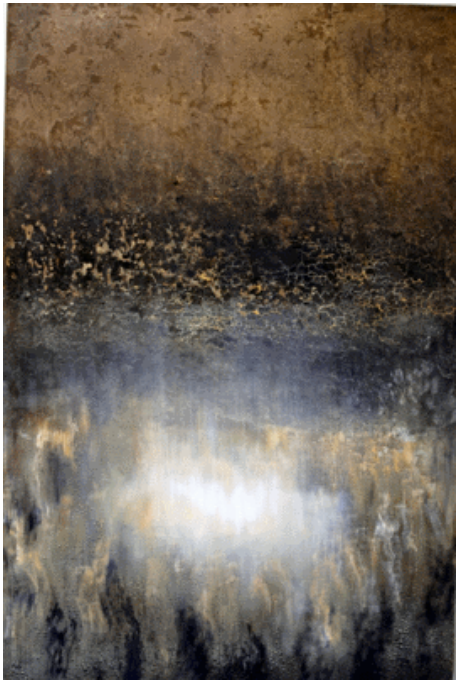

Figure 6. Tracing Truth, 2010 Both paintings are oil and acrylic on canvas, $180 \times 120 \mathrm{~cm}$

Culture and Cosmos 
The next artworks I created were multi-canvases, with multiple gaps and space in between each canvas. The interesting part of this was that each of the paintings was created separately first and then all of them placed independently. Later, all of them became connected with circles and had gaps, as well. Now I expanded the painting as far as I could and removed any odd canvas without interrupting the whole. It was literally a wall basrelief painting with the gaps between the canvases a crucial design element. The canvases and the wall had become a single piece with the gaps as connections. That painting I call Creation. It was only recently completed and took me more than three months to complete. There are 55 canvases in total and as many gaps. I used acrylic and oil.

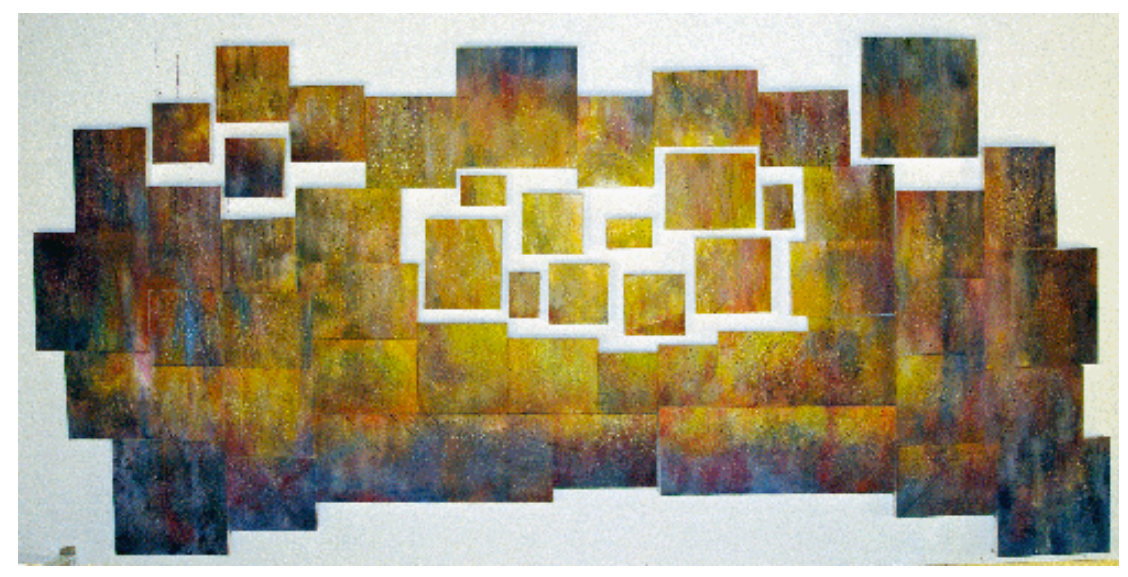

Figure 7. Consciousness:Creation, oil, acrylic on canvas, 375 x $185 \mathrm{~cm}$

I intend my work to suggest an infinite universe, this invisible space portrayed by the visible cloud. This echoes a Hindu proverb that says: 'God is in everything living and non-living, in the earth or the sky. His presence is everywhere and nothing is in his absence'. I try to paint that god of the gap, this space, this emptiness or dark matter. 